\title{
Versuche zur Aufklärung der molekularen Struktur der Thylakoidmembran
}

\author{
Wilhelm Menke \\ Botanisches Institut der Universität zu Köln \\ (Z. Naturforschg. 20 b, 802-805 [1965] ; eingegangen am 12. März 1965)
}

\begin{abstract}
Thylakoids from chloroplasts of Antirrhinum majus and of the blue-green alga Oscillatoria chalybea form folds during the drying on support films. Under favourable conditions, negative staining with uranylacetate gives electron micrographs of these folds which correspond to crosssections of thylakoids. In these preparations the thylakoid membrane seems to consist of a monolayer of particles. These are insoluble in organic solvents. The center to center distance of the particles approximately agrees with the roentgenographically determined center to center distance of the protein particles in the thylakoid membrane. It was not possible to demonstrate lipids in the thylakoid membrane. Because of the background structure of the support film, electron micrographs gave no unequivocal results concerning the structure of the protein layer in surface view.
\end{abstract}

Die bei der Photosynthese photochemisch wirksamen Lamellensysteme bestehen, wie elektronenmikroskopische Untersuchungen zeigten, aus in sich geschlossenen Doppelmembranen, den Thylakoiden ${ }^{1}$. Die einzelne Thylakoidmembran gibt in Querschnitten bei hinreichender Auflösung das Bild einer Elementarmembran nach RoBertson ${ }^{2,3}$; sie scheint demnach symmetrisch gebaut zu sein. Röntgenographische Untersuchungen führten jedoch zu der Auffassung, daß die Thylakoidmembran asymmetrischen Bau besitzt und daß im Thylakoid zwei asymmetrische Thylakoidmembranen spiegelbildlich zueinander orientiert sind ${ }^{4}$. Die Thylakoidmembran ist asymmetrisch, weil sie aus einer homogenen und einer inhomogenen Schicht besteht. Die inhomogene Schicht begrenzt das Thylakoid nach außen und wird aus Partikeln gebildet, die den Röntgenstrahl wie Kugeln von etwa $35 \AA$ streuen ${ }^{5}$. Da das Lamellarsystem der Chloroplasten etwa gleiche Volumanteile Lipide und Proteine enthält, wurde angenommen, daß die homogene Schicht aus Lipiden und die Partikelschicht aus Proteinen besteht. Schon früher hatten polarisationsoptische und volumetrische Untersuchungen wahrscheinlich gemacht, daß Lipide und Proteine in getrennten Schichten nebeneinander liegen ${ }^{1,4}$. Diese Vorstellung vom Bau des Thylakoids darf nun aber nicht als endgültig angesehen werden.

1 Zusammenfassende Darstellungen: W. Menke, Ann. Rev. Plant Physiol. 13, 27 [1962]; „Photosynthesis Mechanisms in Green Plants", Publ. 1145, Natl. Acad. Sci. - Natl. Res. Counc., 537. Washington 1963; Ber. dtsch. bot. Ges. 77, 340 [1964].

2 P. Sitre, Portugaliae acta biolog. [Lisboa] 6, 269 [1962].

3 M. Jost, Arch. Mikrobiol. 50, 211 [1965].
Sie wird in Zukunft schon deswegen modifiziert werden müssen, weil sie die intermolekularen Wechselwirkungen zwischen Lipiden und Proteinen nicht berücksichtigt. Es gibt aber auch experimentelle Befunde, die Zweifel daran aufkommen lassen, ob das derzeitige Modell überhaupt die molekulare Struktur des Thylakoids in ihren Grundzügen zutreffend angibt. So sollen nach PARK und Biggins die als Quantasomen bezeichneten, verhältnismäßig großen Struktureinheiten im Inneren des Thylakoids liegen ${ }^{6}$, was von Jost ${ }^{3}$ mit Hilfe der Gefrierätztechnik bestätigt wird. Daher ist es notwendig, weitere Informationen über die Struktur des Thylakoids zu sammeln, was auf verschiedenen Wegen möglich ist. Da mit der von Brenner und Horne ${ }^{7}$ angegebenen Negativfärbung bei der Aufklärung von molekularen Strukturen beachtliche Erfolge erzielt wurden, lag es nahe, dieses Verfahren auch zur weiteren Aufklärung der Thylakoidstruktur heranzuziehen. Im folgenden wird über die bisher mit diesem Kontrastierungsverfahren erzielten Ergebnisse berichtet.

\section{Methoden}

Chloroplasten wurden aus Blättern von Antirrhinum majus (Sippe 50) durch fraktioniertes Zentrifugieren der wäßrigen Extrakte und durch Zentrifugieren über

4 W. Kreutz u. W. Menke, Z. Naturforschg. 17 b, 675 [1962]. 5 W. Kreutz, Z. Naturforschg. 18 b, 567, 1098 [1963] ; 19 b, 441 [1964].

${ }^{6}$ R. B. Park u. J. Biggins, Science [Washington] 144, 1009 [1964].

7 S. Brenner u. R. W. Horne, Biochim. biophysica Acta [Amsterdam] 34, 103 [1959]. 
einem Dichtegradienten aus Saccharoselösungen isoliert. Zur Entfernung der löslichen Proteine wurde anschließend mit dest. Wasser gewaschen. Untersucht wurden in erster Linie Chloroplastenfragmente, die durch fraktioniertes Zentrifugieren aus den gewaschenen Chloroplasten gewonnen wurden. Die Chloroplastenfragmente wurden $1 / 2$ bis $2 \mathrm{Stdn}$. mit Osmiumtetroxyd (1\%), Formalin (3\%), Glutardialdehyd (5\%) oder Sublimat (1\%) fixiert und anschließend mit dest. Wasser gewaschen. In einigen Versuchen wurden auch Chloroplasten verwendet, die aus Blättern isoliert wurden, die mit Osmiumtetroxyd fixiert worden waren.

Zur Isolierung der Blaualgenthylakoide wurde Oscillatoria chalybea in $m / 15$-Phosphatpuffer $\left(p_{\mathrm{H}} 7,0\right)$ in einem Glasperlenhomogenisator nach $\mathrm{Merken-}$ s chla ger unter Kühlung mit Kohlensäureschnee homogenisiert. Das Homogenat wurde durch eine Glasfritte filtriert und lief dabei in eine 2-proz. Lösung von Osmiumtetroxyd. Die fixierten Thylakoide wurden nach $1 / 2$ bis 1 Stde. durch fraktioniertes Zentrifugieren in dest. Wasser erhalten. Unfixierte Thylakoide wurden aus dem Homogenat durch Zentrifugieren isoliert. Die Arbeitsvorschrift für die Isolierung von Thylakoiden aus Blaualgen wird demnächst von Herrn R. Schmirz veröffentlicht.

Als Objektträgerfolien dienten entweder KohlenstoffFolien, die durch Aufdampfen von Kohlenstoff auf frischgespaltenen Glimmer hergestellt wurden, oder es wurden Kollodiumfolien verwendet, auf die eine dünne Schicht Kohlenstoff aufgedampft worden war.

Zur Herstellung der elektronenmikroskopischen Präparate wurde ein Tropfen einer fixierten oder unfixierten Thylakoidsuspension auf die Kohlenstoff-Folie aufgetragen. Nach etwa 1 Min. wurde der größte Teil der Flüssigkeit mit einem Streifen Filterpapier abgesaugt und sofort ein Tropfen einer 1-proz. Lösung von Uranylacetat $\left(p_{\mathrm{H}} 7,0\right)$ in Wasser zugesetzt. Nach kurzer Zeit wurde der größte Teil der Uranylacetatlösung abgesaugt. Dieses Verfahren gab mit Thylakoiden die besten Ergebnisse. Das Aufsprühen einer Suspension von Thylakoiden in der Lösung des Kontrastmittels bewährte sich weniger. Außer Uranylacetat wurde gelegentlich Phosphorwolframat zur Kontrastierung verwendet. Extraktionsversuche mit organischen Lösungsmitteln wurden entweder auf der Folie vor der Kontrastierung oder vor dem Auftragen vorgenommen.

Die meisten Aufnahmen wurden mit einem Elmiskop I bei 40000 - bis 80000 -facher Vergrößerung angefertigt. Einige Serien wurden mit einem Elmiskop Ia mit Objektraum-Kühlung von Frau Dr. C. Weichan, Berlin, aufgenommen. Die Vermeidung der Objektverschmutzung bewirkt eine bessere Auflösung feiner Objektstrukturen.

\section{Ergebnisse}

Wenn man einen Tropfen einer wäßrigen Suspension von fixierten oder unfixierten Thylakoiden, die Uranylacetat enthält, auf einer Trägerfolie antrock- nen läßt und bei hinreichend hoher elektronenoptischer Vergrößerung aufnimmt, so sollte sich die feinere Struktur der Proteinschicht der Thylakoidmembran sichtbar machen lassen. Die so erhaltenen Aufnahmen (Abb. $1^{*}, 2$ ) entsprechen jedoch nicht den Vorstellungen, die man sich auf Grund der röntgenographischen Untersuchungen gemacht hatte. Man erwartete das Bild einer Schicht aus isodiametrischen oder nahezu isodiametrischen Partikeln, deren Mittelpunktsabstand $35-40 \AA$ beträgt, wobei die Partikel in kleinen Bereichen nach einem quadratischen Gitter geordnet sein sollten. Nach der in Abb. 1 wiedergegebenen Aufnahme scheint die Thylakoidmembran aber aus anisodiametrischen, fibrillären Teilchen aufgebaut zu sein, deren Mittellinienabstand dort, wo sie parallel zueinander verlaufen, etwa $20 \AA$ beträgt. Der scheinbare Durchmesser der Fibrillen entspricht mit etwa $10 \AA$ ungefähr dem Durchmesser der $\alpha$-Helix. Auf verschiedenen Aufnahmen ließ sich entlang der feinsten Fibrillen eine Periodizität von etwa $10 \AA$ mehrfach nachweisen. Diese wird nicht durch die körnige Struktur der Photoschicht vorgetäuscht, da sie bei wiederholter Aufnahme derselben Präparatstelle mit erneuter Scharfstellung unverändert auftrat. Abb. $1 \mathrm{a}$ ist höchstens sehr geringfügig unterfokussiert und sollte daher die Struktur der Thylakoidmembran sowie die Größe und Abstände der Strukturelemente unverzerrt wiedergeben. Bei stärkerer, aber immer noch geringer Unterfokussierung nimmt der Kontrast zu, und es wird in kleinen Bereichen eine eher netzartige Struktur sichtbar (Abb. 2 a). Es können nunmehr mittlere Abstände gemessen werden, die den röntgenographisch ermittelten näherkommen. Um die Gefahr einer Strukturänderung durch Uranylacetat auszuschalten, wurden Aufnahmen von Präparaten angefertigt, die mit Osmiumtetroxyd fixiert waren, jedoch nicht mit Uranylacetat kontrastiert wurden. Überraschenderweise zeigten auch diese Aufnahmen das Bild negativ gefärbter Präparate und ähnliche Strukturen wie die mit Uranylacetat negativ gefärbten Präparate (Abb. 3 a). Außerdem wurden nicht fixierte, jedoch negativ gefärbte Präparate aufgenommen. Selbst die Extraktion der Lipide vor der Negativfärbung änderte die Struktur nicht wesentlich. Der Kontrast war nach Behandlung mit Osmiumtetroxyd und Uranylacetat am größten. Für die Beurteilung der abgebildeten Strukturen ist es nun aber wichtig, daß präparatfreie Stellen der

* Abbn. 1-8 s. Tafel S. 804 a und b. 
Trägerfolie freilich mit viel geringerem Kontrast eine ähnliche Struktur zeigen wie die Präparate und daß sich diese Struktur mit der Fokussierung in ähnlicher Weise ändert wie die Struktur der Präparate (Abbn. 1 b, 2 b, 3 b). Im Bild des Präparats überlagerte sich also die Struktur des Untergrundes und die Eigenstruktur des Präparates. Wegen der dabei auftretenden schwer zu übersehenden elektronenoptischen Effekte läßt sich der Einfluß des Untergrundes nicht ohne weiteres eliminieren. Auf elektronenoptische Effekte ist auch zurückzuführen, daß ungenaue Fokussierung die Struktur des Bildes und die Maße der Strukturelemente stärker beeinflußt als oben angegebene Vorbehandlungen der Präparate. Daher ist bei der Beurteilung der abgebildeten Strukturen größte Zurückhaltung erforderlich.

In einer Suspension von Chloroplastenfragmenten können Thylakoide durch Flüssigkeitsaufnahme anschwellen. Beim Antrocknen an die Folie entstehen dann häufig Falten. Wenn die Suspension Uranylacetat enthält, so dringt diese ins Innere der Thylakoide ein. Beim Antrocken lagert sich außerdem Uranylacetat von außen bevorzugt entlang der Falten an. Wenn die Falten etwa senkrecht zur Trägerfolie stehen, so erhält man gelegentlich von solchen Präparaten Aufnahmen, die einem Querschnitt durch ein Thylakoid entsprechen. Übersichtlich und verhältnismäßig einfach zu deuten sind Aufnahmen von Thylakoiden von Oscillatoria chalybea, da bei dieser Cyanophycee die Thylakoide einzeln im Plasma der Zelle liegen ${ }^{8}$ und man infolgedessen leicht elektronenmikroskopische Präparate mit einzelnen, getrennt nebeneinander angetrockneten Thylakoiden erhält. Abb. 4 zeigt eine solche Aufnahme. Offenbar ist hier nur eine Thylakoidmembran aufgefaltet, so daß man sicher ist, daß der Raum zwischen den beiden Membranen dem Innern des Thy. lakoids entspricht. In der rechten Hälfte der Aufnahme, wo die Thylakoidmembran senkrecht zur Trägerfolie steht, erkennt man, daß jede Membran aus einer Schicht von Partikeln besteht. Ihr Mittelpunktsabstand innerhalb der Membran schwankt um $40 \AA$, während ihre Dicke $30 \AA$ anscheinend nicht überschreitet. Die Falte ist etwa $140 \AA$ dick. Diesem Wert ist jedoch keine besondere Bedeutung beizumessen, da die Dicke der Falten in weiten Grenzen schwankt. Meistens sind die Falten nur 70 bis

8 W. Menke, Z. Naturforschg. 16 b, 543 [1961].
$90 \AA$ dick. Abb. 5 zeigt eine Falte einer Thylakoidmembran von Antirrhinum majus. Auch hier erkennt man an günstigen Stellen, daß jede Thylakoidmembran aus einer Schicht von Partikeln besteht. Für den Mittelpunktsabstand der Partikel innerhalb der Thylakoidmembran können Werte zwischen 35 und $40 \AA$ gemessen werden. Die Thylakoidmembran ist anscheinend etwas dünner als bei Oscillatoria. Die Dicke der Falte beträgt nur $70 \AA$. Abb. 8 zeigt den Auslauf einer etwas schräg zur Trägerfolie liegenden Falte. An dieser Stelle des Präparats standen zufällig die Höhe der Falte und die Schichtdicke des Kontrastierungsmittels in einem solchen Verhältnis zueinander, daß die Umbiegestelle der Membran abgebildet wurde. Sehr häufig findet man mehrere parallel zueinander verlaufende Falten (Abbn. 6 und 7). In diesem Fall ist nicht zu entscheiden, ob es sich um eine mehrfache Auffaltung einer Thylakoidmembran handelt oder ob beide Membranen eines Thylakoids gleichzeitig aufgefaltet sind. Im allgemeinen sind in diesem Fall je zwei Membranen durch eine etwas dickere Schicht des Kontrastierungsmittels voneinander getrennt. Im übrigen bieten die Aufnahmen mit mehreren parallelen Falten nichts Neues. Die Dicke der Falten beträgt häufig 70 bis $90 \AA$ und überschreitet selten $100 \AA$. Die in den Abbn. 4, 5, 6 und 7 wiedergegebenen Aufnahmen stammen von Präparaten, die mit Osmiumtetroxyd fixiert und mit Uranylacetat kontrastiert wurden. Verwendung von Formalin oder von Glutardialdehyd (Abb. 9) führten nicht zu wesentlichen Änderungen, und selbst nicht fixierte Präparate geben Bilder, die sich kaum von den Aufnahmen fixierter Präparate unterscheiden. Extraktion der Lipide vor der Negativfärbung bewirkt keine wesentlichen Veränderungen. Daher darf man annehmen, daß bei diesem Verfahren nur das Strukturproteid der Thylakoide zur Darstellung gelangt. Da die Dicke einer Falte nicht selten nur $70 \AA$ beträgt, kann die Dicke der einzelnen Proteinschicht der Thylakoidmembran $35 \AA$ nicht überschreiten. Zwischen den Proteinschichten des Thylakoids ist kein Raum für zwei Lipidschichten von je $40 \AA$ vorhanden. Aber auch dort, wo mehrere Membranpaare parallel zueinander verlaufen, ist zwischen den Membranpaaren kaum genügend Raum für zwei Lipidschichten. Da bisher alle Versuche, die Lipide in den Präparaten zur Darstellung zu bringen, fehlschlugen, die Thylakoide aber andererseits etwa $40 \%$ Lipide enthalten, muß man annehmen, daß bei der Auffaltung 


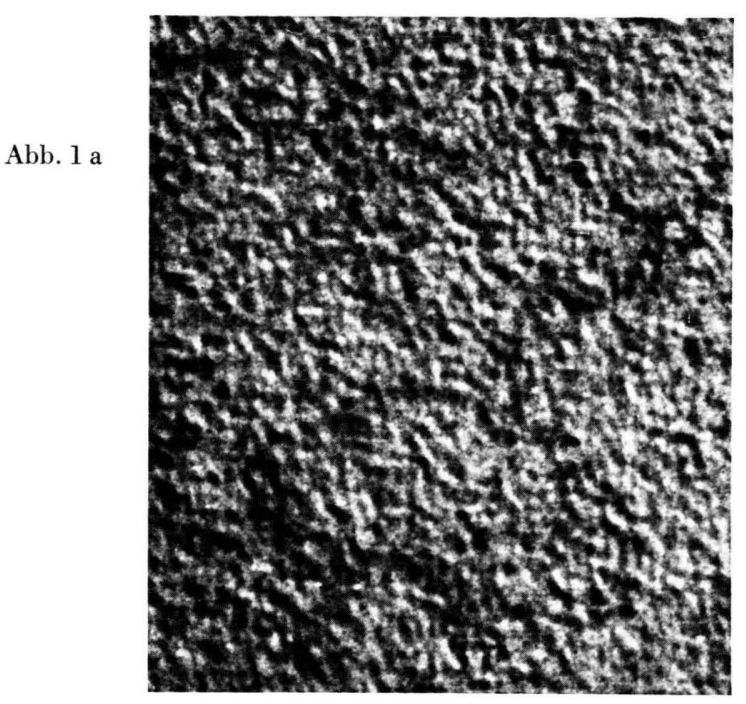

Abb. 2 a

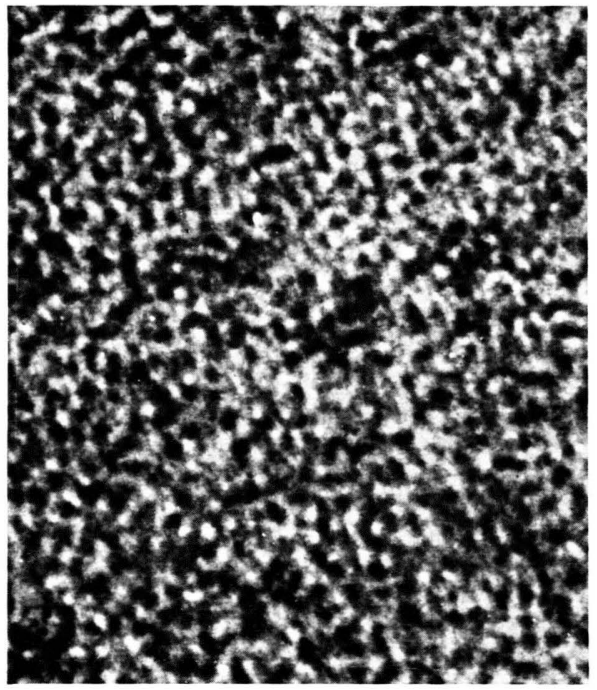

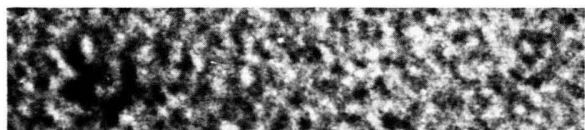

Abb. 3 a

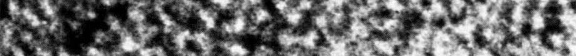

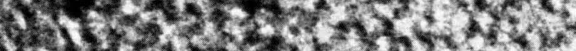

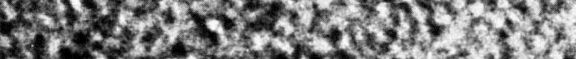

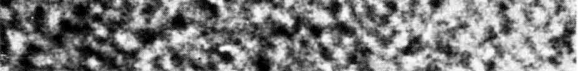

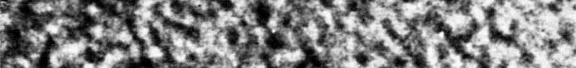

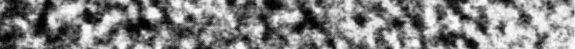

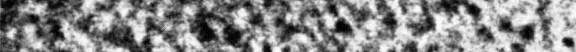
20,

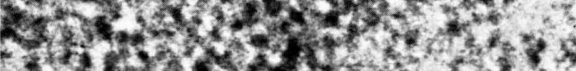

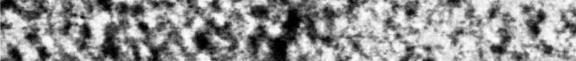

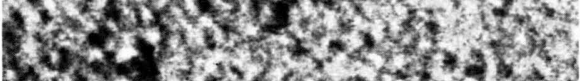

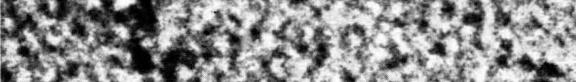
$x^{2}+2 x^{2} y^{*}+x^{2}+21$

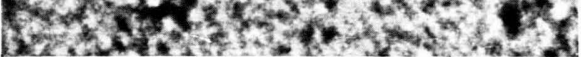

Abb. 1 b

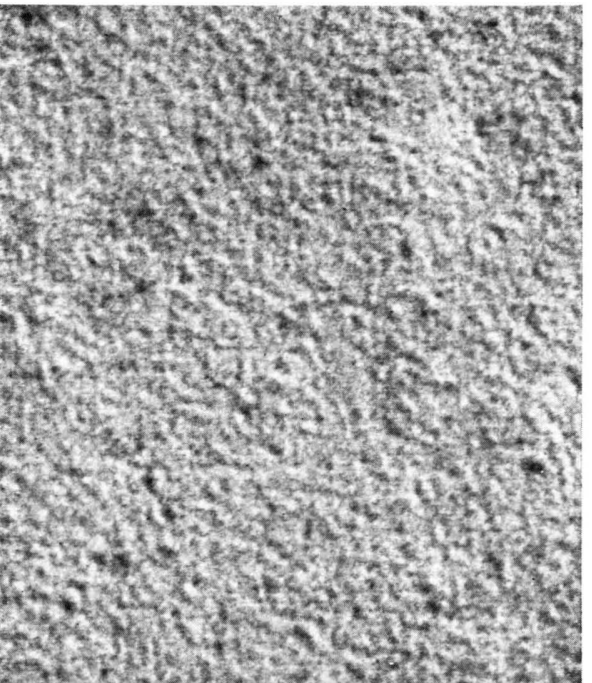

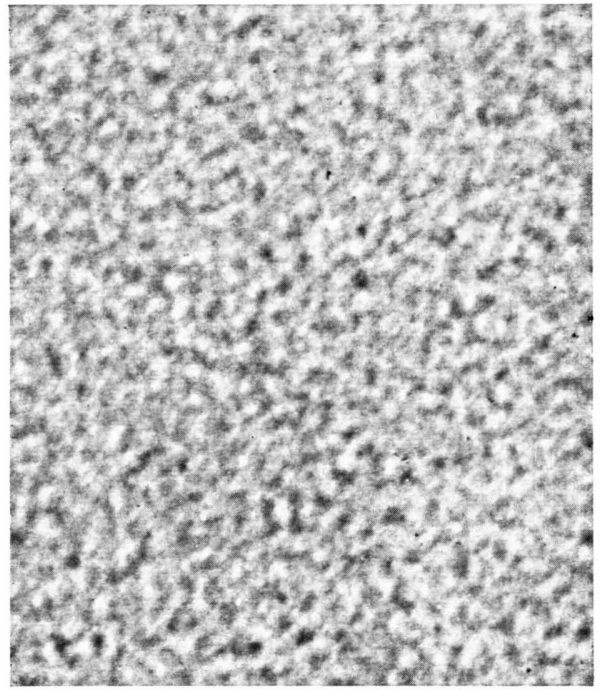

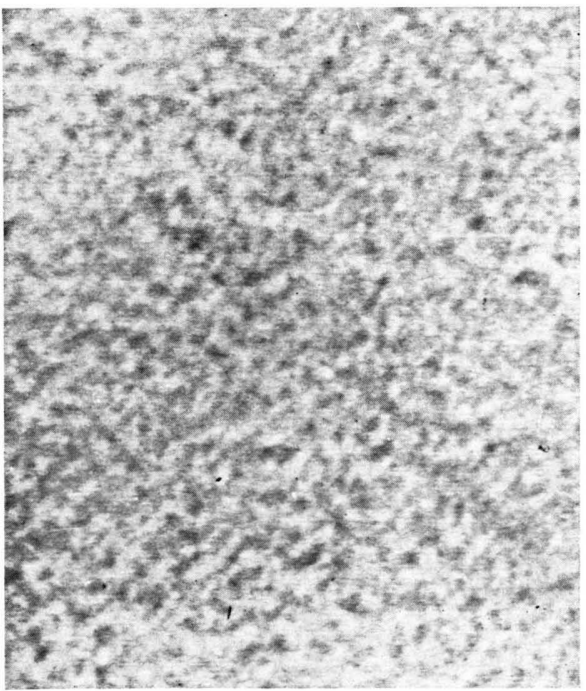

Abb. $3 \mathrm{~b}$ 
Abb. 4

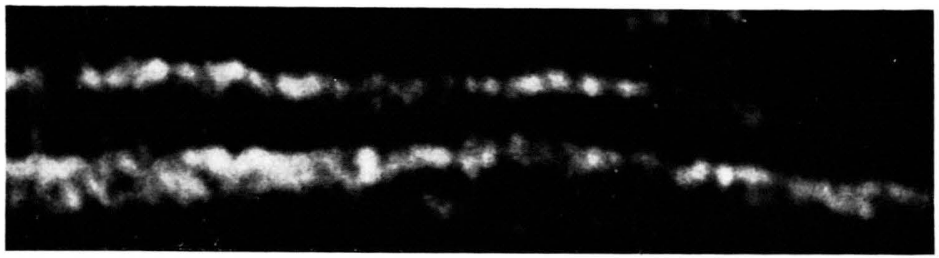

Abb. 5

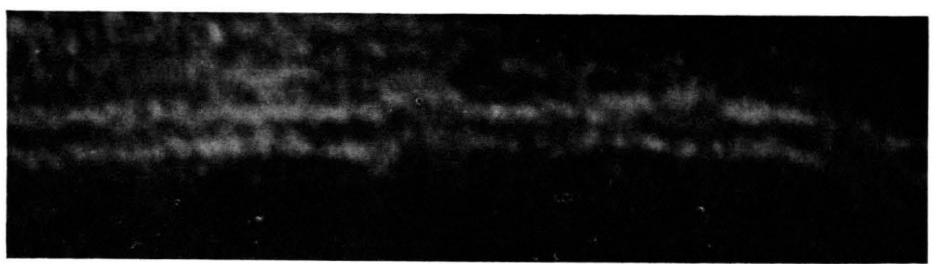

Abb. 6

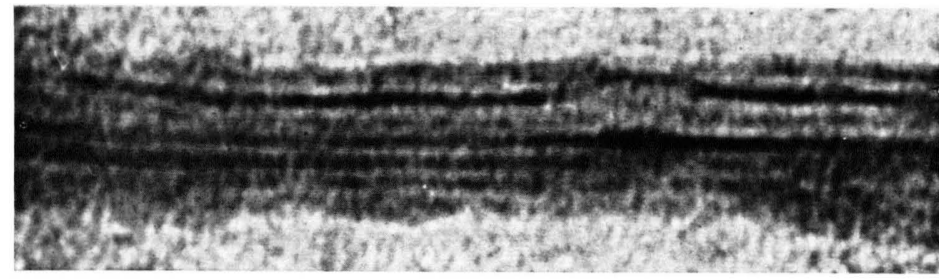

Abb. 7

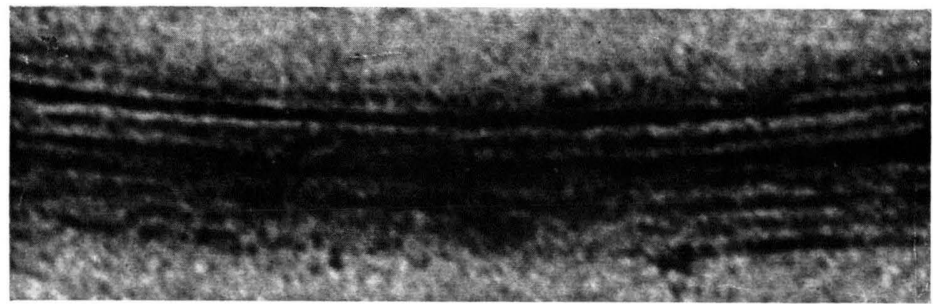

Abb. 8
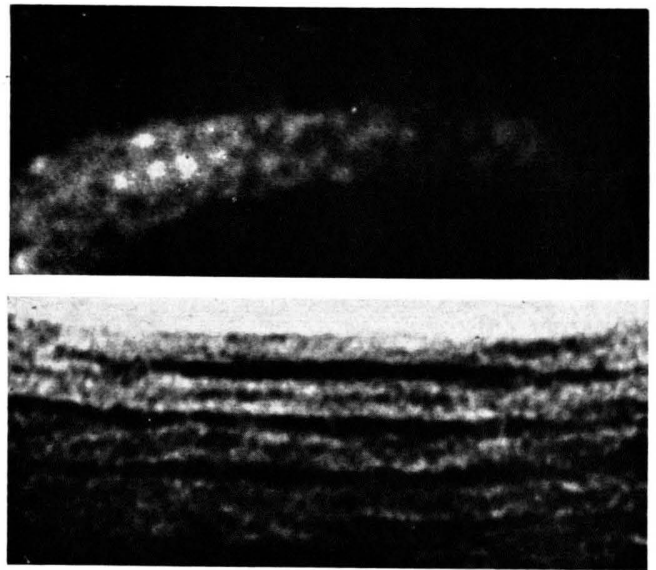

Abbn. 1, 2 und 3. a) Teile von Thylakoiden von Antirrhinum majus in Flächenansicht. b) Objektfreie Stellen der Trägerfolie. 1. Fixierung: Osmiumtetroxyd; Kontrastierung: Uranylacetat. 2. Fixierung: - ; Kontrastierung: Uranylacetat. 3. Fixierung: Osmiumtetroxyd ; Kontrastierung: - . Aufnah nahmen 1 und 3 mit Objektraum-Kühlung (Dr. W WICHAN).

$$
\text { Vergr. } 960000: 1 \text {. }
$$

Abb. 4. Auffaltung einer Thylakoidmembran von Oscillatoria chalybea. Die obere Thylakoidmembran steht etwa senkrecht zur Objektträgerfolie, die untere ist in der linken Hälfte zur Objektträgerfolie geneigt. Fixierung: Osmiumtetroxyd; Kontrastierung: Uranylacetat; Vergr. $690000: 1$.
Abb. 5. Auffaltung einer Thylakoidmembran von Antirrhinum majus. Fixierung: Osmiumtetroxyd; Kontrastierung: Uranylacetat; Vergr. $690000: 1$

Abbn. 6 und 7. Parallel verlaufende Falten in Thylakoiden von Antirrhinum majus. Fixierung: Osmiumtetroxyd; Kontrastierung: Uranylacetat; Vergr. $480000: 1$.

Abb. 8. Auslauf einer Falte einer Thylakoidmembran von Antirrhinum majus. Kontrastierung: Uranylacetat;

$$
\text { Vergr. } 960000: 1 \text {. }
$$

Abb. 9. Falten eines Thylakoids von Antirrhinum majus. Fixierung: Glutardialdehyd; Kontrastierung: Uranylacetat. Vergr. $480000: 1$. 
und beim Trocknen die Lipide zum größten Teil aus den Falten entfernt werden. Keineswegs kann man das Fehlschlagen aller Versuche, die Lipide in den Falten der Thylakoide darzustellen, durch die Annahme erklären, daß sich die Chloroplastenlipide durch Negativfärbung nicht darstellen ließen. Myelinfiguren aus extrahierten Chloroplastenlipiden zeigen nach Negativkontrastierung einen Schichtenbau mit einer Periode von etwa $50 \AA$. Die Untergrundstruktur wirkt sich auf die Abbildung der Falten viel weniger aus als auf die Abbildung der Thylakoide von der Fläche her. Wegen der größeren Unterschiede zwischen den Massendicken von Objekt und Kontrastierungsschicht darf der Einfluß des Untergrundes bei der Deutung der Aufnahmen von Falten zunächst unbeachtet bleiben. Infolge der größeren Dicke der Präparate wird die Ermittlung der realen Struktur jedoch durch die Überlagerung von mehreren Strukturelementen erschwert. Die auf den elektronenmikroskopischen Aufnahmen gemessenen Partikelabstände und Partikelgrößen sind auch hier um einen Faktor $1 / 2$ bis 2 unsicher. Die beiden wichtigsten Ursachen für diese Unsicherheit sind unexakte Fokussierung und Überlagerungseffekte.

So kann man mit einigen Vorbehalten aus diesen Untersuchungen nur den Schluß ziehen, daß jede Thylakoidmembran eine Proteinschicht enthält, die aus diskreten, nebeneinander liegenden Partikeln besteht. Dabei stimmt die mittlere Periode in der Ebene der Proteinschicht innerhalb der verhältnismäßig hohen Fehlergrenzen mit den früher röntgenographisch ermittelten Werten überein. Die Gestalt der Partikel und ihre Ordnung in der Ebene der Proteinschicht bleiben ungewiß. Es erscheint jedoch zweifelhaft, daß die Proteinschicht aus nahezu isodiametrischen Teilchen besteht. Mit dem ursprünglichen Modell der Elementarmembran sind die

9 S. E. G. Nilsson, J. Ultrastructure Res. 11, 581 [1964] ; 12, 207 [1965]. bisher mit Hilfe der Negativkontrastierung erzielten Ergebnisse nicht in Einklang zu bringen, da dieses Modell zwei Proteinschichten enthält. Neuere elektronenmikroskopische Untersuchungen von NiLssoN $^{9}$ an Schnittpräparaten, bei denen eine höhere Auflösung als bisher erzielt wurde, führen jedoch ebenfalls zu Vorstellungen, welche mit der bisherigen Auffassung vom Bau der Elementarmembran nicht recht übereinstimmen. Die Elementarmembran in den Außengliedern der Stäbchen der Retina werden bei hoher Vergrößerung als dunkle Bänder abgebildet, in deren Mitte eine Reihe heller, etwa kreisförmiger Gebilde liegt. Ähnliche Bilder hatte SJöstrand ${ }^{10}$ schon früher von Mitochondrienmem. branen veröffentlicht. Abstände und Abmessungen dieser Gebilde stimmen etwa mit den hier in der Thylakoidmembran abgebildeten Partikeln überein. Die chemische Natur dieser Partikel läßt NiLsson ${ }^{9}$ offen. In der Thylakoidmembran handelt es sich allem Anschein nach um Proteinpartikel. Wenn diese Deutung zutrifft, muß man freilich annehmen, daß nach Fixierung mit Osmiumtetroxyd die Proteinpartikel in Membranen, die aus Lipiden und Proteinen bestehen, mit negativem Kontrast abgebildet werden können. Für das Zutreffen dieser Annahme geben auch die vorliegenden Untersuchungen einen Hinweis.

Die Arbeit wurde von der Deutschen For schung g e meins chaft unterstützt. Ich danke Frau Dr. C. Weichan, Berlin, für die Anfertigung einiger Aufnahmeserien mit dem Elmiskop Ia mit Objektraum-Kühlung. Zu Dank bin ich ferner Herrn R. Schmitz für die Überlassung von Blaualgenkulturen, Fräulein E. Schölzel für die Herstellung von Präparaten und Herrn G. Pesch für die Ausführung der photographischen Arbeiten verpflichtet. Bei der Klärung verschiedener Fragen waren mir Diskussionen mit Herrn Dr. L. Diers von großem Nutzen.

10 F. S. Sjöstrand, J. Ultrastructure Res. 9, 340 [1963]. 